\title{
Quantum integrability of perturbed Virasoro-degenerate conformal models
}

\author{
M. Niedermaier \\ Institute for Theoretical Physics, University of Hamburg, Luruper Chaussee 149, D-2000 Hamburg 50, FRG
}

Received 13 March 1990

Off-critical conservation laws of a class of irrational conformal models are examined. It has been conjectured that the massive theories that arise under perturbation with the energy (respectively spin-density) operator possess an infinite number of conservation laws, constructable in the Hilbert space of the CFT. A constructive proof of this conjecture is given by means of an algorithmic Fock space procedure.

A distinctive feature of 2-dimensional conformal field theory (CFT) is the clear-cut factorization of the theory into right- and left-moving lightcone sectors. To a large extent the solution of a CFT can then be traced back to the representation theory of infinite dimensional Lie algebras. Whenever such a factorization cannot be achieved a priory, i.e. left-and right-movers mix in an essential way, most of the stringent algebraic structure seems to be lost. As part of his program [1-4] to study the scaling region around a CFT, Zamolodchikov proposed in ref. [5] that the off-critical behavior of certain CFTs, perturbed by suitable relevant operators, should be governed by an infinite number of conserved currents of higher spin $N \geqslant 2$. The latter can be taken as a pragmatic definition of integrability. These currents are solutions of the compatibility relation for the mapping

$\partial_{\bar{z}}=\partial_{\bar{z}}(P, Q): \hat{\mathscr{H}}_{P} \rightarrow \hat{\mathscr{H}}_{Q} \otimes \hat{\mathscr{H}}_{Q}, \quad\left(\partial_{\bar{z}} P\right)(z, \bar{z})=\oint_{z} \mathrm{~d} u \phi_{Q}(u, \bar{z}) P(z)$

and its antiholomorphic partner

$\partial_{\bar{z}}(P, Q) P_{N}=\partial_{z} Q_{N-2}=\partial_{\bar{z}} Q_{N-2}=\partial_{z}(\ddot{P}, Q) \bar{P}_{N}$.

Here $\phi_{Q}$ is the perturbation operator and $\hat{\mathscr{H}}_{P}, \hat{\mathscr{H}}_{Q}$ are representation spaces of the chiral algebra of the (left-right symmetric) CFT in question (with total derivatives divided out; $P$ is usually the vacuum representation, since then intermediate representation are absent). These currents, having integer spin, are local in the CFT, but will be highly non-local in the perturbed theory. In the superrenormalizable regime now, the Hilbert space of the resulting massive theory is expected to be still that of the CFT - and hence a representation space of their chiral algebra. Some recent references in this direction are refs. [6,7]. This additional algebraic framework would then hopefully allow one to (partially) replace the lattice regularization of the quantum inverse scattering method, which often spoils the integrability of the classical model on starts with. If so, the chance of getting a hand on the exact solution of a large class of massive field theories would be considerably increased. Up to now, neither the existence of the currents (2), nor their relation to a more conventional basis (e.g. asymptotic states) of the massive Hilbert space seem to have been established. In this letter, we will address the first question for a particular class of irrational conformal models, parametrized by a Lie algebra [here $\mathrm{Sl}(2), \mathrm{Sl}(3)$ ], and a real parameter $\alpha_{+}^{2}$, related to the central charge. Following the suggestion of ref. [8] [for $\mathrm{Sl}(2)$ ], we will call these models (extended) Dotsenko-Fateev (DF) models. For rational values of $\alpha_{+}^{2}$ they reduce to the (extended) minimal 
models of refs. [9,10]. Although irrational, many of the standard techniques of CFT can still be applied [8,11]. In the present context, they are particularly suited to study genuine off-critical effects, without the additional complication that arises from the rich null-vector structure of the minimal models.

For the unextended irrational models, Zamolodchikov considered in ref. [5] the perturbation by the $\phi_{31}$ "energy density" \#1 and $\phi_{21}$ "spin density" operator and conjectured the existence of an infinite number of conserved currents at grades

$N=2 k, k \in \mathbb{N}, \quad$ for $\phi_{Q}=\phi_{(31)}$ and $\frac{1}{4} \leqslant \alpha_{+}^{2} \leqslant 1$,

$N=6 k+2,6 k, k \in \mathbb{N}$ for $\phi_{Q}=\phi_{(21)}$ and $\frac{1}{6} \leqslant \alpha_{+}^{2} \leqslant 2$.

The range of the screening charge is chosen respectively such that $\phi_{Q}$ is the most relevant field of an operator product subalgebra it belongs to. A proof of the first conjecture will be given below. A proof of the $\phi_{21}$ case can be obtained by embedding the Verma module into the Fock module of an Sl(3)-extended DF model. One first shows that under perturbation with the Sl(3) analogue $\phi_{(22)(11)}$ of the $\phi_{(31)}$ operator, the extended DF model possesses an infinite number of conserved currents at grades $\# 2$

$N=2,3 \bmod 3$ for $\phi_{(22)(11)}$.

This is done by characterizing the conserved densities as Weyl invariant, quasiprimary Fock space solutions of the differential equations

$\left(\mathscr{V}_{\alpha_{+}}^{i}\right)_{-1} P_{N}=0, \quad i=1,2$

where $\left(\mathscr{V}_{\alpha_{+}}^{i}\right)_{-1}$ are the residues of the screening operators of the Sl(3) DF model. In this situation, a version of the recursive argument given below for the case (a) can be applied. The result for the $\phi_{21}$ perturbation of the unextended model can then be obtained by a truncation. Although we have basically completed this construction, there are a number of subtleties involved, that require a more detailed exposition than can be given here (see ref. [12]). For the rest of this letter we will restrict attention to the proof of case (a).

The Hilbert space of an (unextended) DF model is a direct sum of irreducible representation spaces of a pair of Virasoro algebras:

$\mathscr{H}=\underset{M}{\bigoplus} \mathscr{H}_{M} \otimes \overline{\mathscr{H}}_{M}$.

The sum runs over all completely degenerate highest weight representations of Vir with the given central charge $c=13-6 \alpha_{+}^{2}-6 \alpha_{+}^{-2}$. These are labelled by a pair of integers $M=\left(m^{\prime}, m\right)$. The highest weight is given by $h_{m^{\prime} m}=h\left(\alpha_{m^{\prime} m}\right) ; \quad h(\alpha)=\frac{1}{2} \alpha\left(\alpha-4 \sqrt{2} \alpha_{0}\right), \quad 2 \alpha_{0}=\alpha_{+}+\alpha_{+}^{-1}, \quad$ where $\quad \alpha_{m^{\prime} m}=(1 / \sqrt{2})\left(1-m^{\prime}\right) \alpha_{+}+(1 /$ $\sqrt{2})(1-m) \alpha_{+}^{-1}$ is a weight vector of $\mathrm{Sl}(2)$. As usual, $\mathscr{H}_{M}$ is obtained as the quotient of the Verma module with highest weight $h_{M}$ by this maximal singular submodule $\mathscr{H}_{M}=V_{M} / S V_{M}$. For $\alpha_{+}^{2}$ irrational $S V_{M}$ is generated by a single null-vector at level $m^{\prime} m$. This allows a simple Fock space construction of $\mathscr{H}_{M}$ : Introduce the Fock module of a single free boson

$F_{\alpha, \alpha_{0}}=\bigoplus_{\lambda} a_{-\lambda} v_{\alpha, \alpha_{0}}$,

$\left[a_{n}, a_{m}\right]=n \delta_{n+m, 0} \quad a_{n} v_{\alpha, \alpha_{0}}=0, \quad n>0, \quad a_{0} v_{\alpha, \alpha_{0}}=\alpha v_{\alpha, \alpha_{0}}, \quad v_{\alpha, \alpha_{0}}=d_{\alpha} v_{0, \alpha_{0}}$.

Here and in the sequel, we index the states of a module by partitions $\lambda=\left(\lambda_{1}, \ldots, \lambda_{l(\lambda)}\right), 1 \leqslant \lambda_{1} \leqslant \ldots \leqslant \lambda_{p} ; l(\lambda)$ is called the length of the partition and $a_{-\lambda}$ is shorthand for $a_{-\lambda_{1}} a_{-\lambda_{2}} \ldots a_{-\lambda_{p}}$. The Feigin-Fuks construction of nullvectors [13] gives the kernel of the canonical projection

\#1 The completely degenerate representations of a Sl $(r+1)$ DF model are labelled by pairs of $\mathbb{Z}^{r}$ vectors. We will always take $P$ to be the vacuum representation, $P=(11)$, etc.

\#2 Here there are subcases for the range of $\alpha_{+}^{2}$, because $\phi_{(22)(11)}$ is contained in more than one subalgebra [12]. 
$\phi_{m^{\prime} m}: V\left(h_{m^{\prime} m}, c\right) \rightarrow F_{m^{\prime} m}, \quad L_{-\lambda}\left|h_{m^{\prime} m}\right\rangle \rightarrow \sum_{\mu}\left(\phi_{m^{\prime} m}\right)_{\lambda \mu} a_{-\mu} v_{m^{\prime} m}$

$L_{-n}=\left[a_{0}+(n-1) \sqrt{2} \alpha_{0}\right] a_{-n}+\frac{1}{2} \sum_{k=1}^{n-1} a_{-k} a_{k-n}+\sum_{k \geqslant 0} a_{-n-k} a_{k}, \quad n>0$.

In case of a single null-vector it is directly given by the image of

$Q_{m}: F_{-m^{\prime}, m} \rightarrow F_{-m^{\prime},-m}, \quad m^{\prime}, m>0, \quad Q_{m}=\int \mathrm{d} u_{1} \ldots \mathrm{d} u_{m} V_{\alpha_{+}}\left(u_{1}\right) \ldots V_{\alpha+}\left(u_{m}\right)$,

with $V_{\alpha_{+}}(z)$ the screening operator. Thus

$\mathscr{H}_{m^{\prime} m} \simeq F_{m^{\prime} m} / \operatorname{Ker} \phi_{m^{\prime} m} \simeq F_{m^{\prime} m} / Q_{m} F_{-m^{\prime}, m}$

and employing duality ( $F_{m^{\prime} m}^{*} \cong F_{-m^{\prime},-m}$ as Verma modules; $Q_{m}^{\dagger}=Q_{m}$ ) one has the physical state condition [8]

$\mathscr{H}_{m^{\prime} m} \cong \operatorname{Ker}\left(Q_{m}: F_{m^{\prime} m} \rightarrow F_{m^{\prime},-m}\right), \quad m^{\prime}, m>0$.

For the discussion of conservation laws we further need the notion of a quasiprimary state. Let

$(A B)(z)=\oint_{z} \frac{A(u) B(z)}{u-z}$

denote the normal ordering adopted to the operator product expansion, with $A(z)=\sum_{n} A_{n} z^{-n-a}$, $B(z)=\sum_{n} B_{n} z^{-n-b}$ momentarily two fields of integer spin. It gives a natural 1-1 correspondence between rightnested operator monomials and states in the corresponding module (based on a vacuum $v$ ):

$$
\begin{aligned}
& \lim _{z \rightarrow 0}\left(A^{n_{1}-a}\left(A^{n_{2}-a}\left(\ldots A^{n_{k}-a}\left(B^{m_{1}-b}\left(B^{m_{2}-b}\left(\ldots B^{m_{l}-b}\right) \ldots\right)\right) \ldots\right)\right)\right)(z) v \\
& \quad=\left(n_{1}-a\right) !\left(n_{2}-a\right) ! \ldots\left(n_{k}-a\right) !\left(m_{1}-b\right) !\left(m_{2}-b\right) ! \ldots\left(m_{l}-b\right) ! A_{-n_{1}} A_{-n_{2}} \ldots A_{-n_{k}} B_{-m_{1}} B_{-m_{2}} \ldots B_{-m_{l}} v .
\end{aligned}
$$

The inverse mapping is given by

$\left(A_{-n} B_{-m}\right)(z)=\oint_{z} \mathrm{~d} u(u-z)^{a-n+b-m-1} A(u) B(z)$, etc.

This is designed such that the action of $\partial_{z}$ on operators coincides with that of $L_{-1}$ on states. The equivalence classes modulo $L_{-1}$ exact pieces shall be called quasiprimary states ${ }^{\# 3}$ and the corresponding modules signified by a hat, e.g. $\hat{V}_{M}=V_{M} / L_{-1} V_{M}$, etc. Forgetting about the antiholomorphic part, we are interested in the kernel of

$\partial_{z}: \hat{H}_{11} \rightarrow \hat{H}_{31}, \quad \partial_{z}=\left(\mathscr{V}_{\alpha_{31}}\right)_{-1} d_{\alpha_{31}}$.

Here we have split off the charge-shifting part of the vertex operator and adopted a non-standard moding:

$$
\begin{aligned}
& V_{\alpha}(z)=d_{\alpha} z^{a a 0} \mathscr{V}_{\alpha}(z), \\
& \mathscr{V}_{\alpha}(z)=\exp \left(\sum_{n \geqslant 1} \frac{\alpha a_{-n} z^{n}}{n}\right) \exp \left(\sum_{n \geqslant 1} \frac{\alpha a_{n} z^{-n}}{n}\right)=\sum_{k \in z}\left(\mathscr{V}_{\alpha}\right)_{k} z^{k} \\
& \left(\mathscr{V}_{\alpha}\right)_{k}=\sum_{l \geqslant 0} h_{l+k}\left(\alpha a_{-n} / n\right) h_{l}\left(-\alpha a_{n} / n\right),
\end{aligned}
$$

$h_{k}$ being the elementary Schur polynomials. From

*3 The usual definition $L_{1} \mid>=0[7]$ fixes a representative. 
$\left(\mathscr{V}_{\alpha}\right)_{k} v_{\alpha, \alpha_{0}}=h_{k}\left(\alpha a_{-n} / n\right) v_{\alpha, \alpha_{0}}=\frac{1}{k !}\left(L_{-1}^{(a)}\right)^{k} v_{\alpha, \alpha_{0}}$

one has coincidence with the Verma module formulation [5].

On the vacuum Fock module, the physical state conditions $(12)$ is $\mathscr{H}_{11} \cong \operatorname{Ker}\left[\left(\mathscr{V}_{\alpha_{+}}\right)_{-1}\right]$. Since $\mathscr{V}_{31}=\mathscr{V}_{-\alpha+}$ one sees that the conserved densities are characterized as physical, quasiprimary, $S_{2}$ invariant states in $F_{11}$ [14]. Here we defined a physical quasiprimary Fock state as a state $f \in \hat{F}_{\alpha, \alpha_{0}}$ such that there exists another state $g \in \hat{F}_{\alpha, \alpha_{0}}$ with $\left(\mathscr{V}_{\alpha+}\right)_{-1} g \neq 0$ and

$\left(\mathscr{V}_{\alpha_{+}}\right)_{-1} d_{\alpha_{+}}\left(f+L_{-1} g\right)=0$.

$S_{2}$ is the Weyl group of $\mathrm{Sl}(2)$ and acts trivially via $a_{n} \rightarrow(-)^{n+1} a_{n}$ on $F_{\alpha, \alpha_{0}}$. Explicitly, the conserved densities $P_{N}$ can be constructed in two ways. Either one pre-selects the $S_{2}$ invariant Fock monomials of a given grade in an Ansatz for $f$ and then tries to solve eq. (21); or one pre-selects physical quasiprimary Verma module states and tries to find $S_{2}$ invariant combinations of their Fock space projections. Consider for illustration $N=6$, say. Either way one finds (skipping the vacuum $v_{11} ; \simeq$ is the equivalence relation in $\hat{F}_{11}$ )

$P_{6}=L_{-2}^{3}+\left(2 \alpha_{0}^{2}-\frac{1}{4}\right) L_{-3}^{2} \simeq \frac{1}{8} a_{-1}^{6}+5\left(\alpha_{0}^{2}-\frac{1}{2}\right)\left(a_{-1} a_{-2}\right)^{2}+16\left(\alpha_{0}^{4}-\frac{2}{3} \alpha_{0}^{2}+\frac{23}{768}\right) a_{-3}^{2}$

in agreement with ref. [5].

However, the Fock space formulation alone does not gain much in establishing the existence of an infinite set of conservation laws. Taking $f$ in (21) $\mathrm{S}_{2}$ invariant, $g$ arbitrary, one easily checks from the partition functions that (21) amounts to a linear system of 3,7,16,30, 56 equations for 4, 8, 15,30, 55 unknowns at grades $N=4$, $6,8,10,12$ respectively. (The number of quasiprimary Fock monomials of odd power at grade $N$ is $\frac{1}{2}\left[P(N)-P(N-1)-\frac{1}{2}(N-2)\right]$ for $N$ even, where [ ] denotes the integer part and $P(N)$ the number of partitions of $N)$. For $N \geqslant 8$ this system cannot be expected to have solutions on mere dimensional grounds. This means that besides the dimensions of the various subspaces of $\hat{F}_{11}$, a partial input is required about the actual form of the physical states. Fortunately, this input turns out to be very simple, and gives rise to a recursive algorithm by means of which the existence of the conservation laws can be established.

Introduce the Fock module

$\hat{F}_{11}=\operatorname{Ker} Q_{1} /\left(\hat{F}_{11}\right)_{0}$

with the $S_{2}$ invariant sector $\left(\hat{F}_{11}\right)_{0}$ modded out. The vertex operator in (21) maps invariant polynomials onto each other, so that this makes sense. Further one infers that the physical state condition involves mixing of a Fock monomial of power $p$ with all those of lower powers. This means that $P(\leqslant k):=\left.\operatorname{dim} \hat{\mathscr{F}}_{11}\right|_{p \leqslant k}$ is well defined, giving the number of physical quasiprimary states (at a given grade) in which the highest non-invariant monomial has power $k$. Now suppose that at fixed grade $N$ a list of physical, quasiprimary Fock states has been given and one searches for a $S_{2}$ invariant linear combination of them. The above properties of the physical state condition then imply that if one does this by elimination in order of decreasing power, one can use the dimensional bounds $P(\leqslant k), k \leqslant N$, contained in the original list. Let $C(\leqslant k)$ denote the number of representatives of $\left.\hat{\mathscr{F}}_{11}\right|_{p \leqslant k}$ constructed in the course of the elimination process. The recursion process is now driven by the fact that after the first step $C(\leqslant k) \geqslant P(\leqslant k)$, for all $k \leqslant N-5$ and the highest monomials of the representatives are forced to lie within a $P(\leqslant k)$-dimensional subspace of $\tilde{F}_{11}$.

The first non-trivial example for the mechanism at work here occurs at grade $N=10$, so consider this for illustration. A basis for the physical quasiprimary Fock states is given by the Fock space projections of the Verma module states. To describe their structure, the following symbolic notation is convenient: Write $[a]^{p}$ for a quasiprimary Fock monomial of power $p$ and let $\left(\alpha_{0}^{k}\right)$ denote a polynomial in $\alpha_{0}$ of maximal power $k^{\sharp 4}$. In this notation, the projections in $\widehat{\mathscr{F}}_{11}$ are of the form

\#4 If $\hbar$ were included, this would be a homogeneous polynomial in $\alpha_{0}$ and $\hbar$. 
$L_{-8} L_{-2} \simeq\left(\alpha_{0}\right)[a]^{3}$,

$L_{-6} L_{-2}^{2} \simeq\left(\alpha_{0}\right)[a]^{5}+\left(\alpha_{0}^{3}\right)[a]^{3}$,

$L_{-4} L_{-2}^{3} \simeq\left(\alpha_{0}\right)[a]^{7}+\left(\alpha_{0}^{5}\right)[a]^{5}+\left(\alpha_{0}^{3}\right)[a]^{3}$,

$L_{-2}^{5} \simeq\left(\alpha_{0}^{3}\right)[a]^{7}+\left(\alpha_{0}^{5}\right)[a]^{5}+\left(\alpha_{0}^{3}\right)[a]^{3}$,

where $\simeq$ is now the equivalence relation in $\hat{\mathscr{F}}_{11}$. The monomials to be eliminated are just those with odd power, and their number at power $p=3,5,7,9$ is respectively given by $1,2,1,0$. Clearly, $[a]^{7}$ can be eliminated trivially, resulting in

$L_{-2}^{5}+\left(\alpha_{0}^{2}\right) L_{-4} L_{-2}^{2} \simeq\left(\alpha_{0}^{5}\right)[a]^{5}+\left(\alpha_{0}^{5}\right)[a]^{3}$.

Since the space of quasiprimary Fock monomials of power 5 is 2-dimensional, the combination occurring in (25) could in principle be different from that in (24b), apparently providing an obstruction to further elimination. However, from (24a) $-(24 \mathrm{~d})$ we know that $P(\leqslant 5)=1$, so that in fact the combinations have to coincide (the author has checked this by calculation). Thus $[a]^{5}$ can also be eliminated resulting in

$L_{-2}^{5}+\left(\alpha_{0}^{2}\right) L_{-4} L_{-2}^{2}+\left(\alpha_{0}^{4}\right) L_{-6} L_{-2}^{2} \simeq\left(\alpha_{0}^{7}\right)[a]^{3}$.

The last step is again trivial giving the conserved density

$P_{10}=L_{-2}^{5}+\left(\alpha_{0}^{2}\right) L_{-4} L_{-2}+\left(\alpha_{0}^{4}\right) L_{-6} L_{-2}^{2}+\left(\alpha_{0}^{6}\right) L_{-8} L_{-2}$.

In retrospect note that, say in the second elimination step, the information $\left.\operatorname{dim} \hat{\hat{F}_{1}}\right|_{N=10, p=5}=2$, is actually not needed. The new state with highest non-invariant term at $p=5$ is forced to lie within the $P(\leqslant 5)$-dimensional subspace $\left.\tilde{\mathscr{F}}_{11}\right|_{p \leqslant 5}$. Also the value $P(\leqslant 5)$ is not needed. In any case, one has $P(\leqslant 5)+1$ representatives of the $P(\leqslant 5)$ equivalence class, so that the linear system representing the elimination has at least one solution. This drives the next recursion step, etc.

The result for generic $N$ follows now from the facts: for $N$ even, $N \geqslant 6$,

(1) $\left.\operatorname{dim} \hat{\mathscr{F}}_{11}\right|_{N, p}>1 \quad$ iff $3 \leqslant p \leqslant N-4,\left.\quad \operatorname{dim} \hat{F}_{11}\right|_{N, N-1}=0, \quad P(\leqslant N-3)=2$

(2) $\left.\operatorname{dim} \hat{\mathscr{H}}_{11}\right|_{N, l}=P(\leqslant 2 l-1), \quad l \leqslant N / 2-1 \quad\left[l:=l(\lambda)\right.$ in $L_{-\lambda} ; p:=l(\lambda)$ in $\left.a_{-\lambda}\right]$.

Point (2) express the fact that there is no need to solve the combinatorial problem of calculating the absolute values of the numbers $P(\leqslant k), 1 \leqslant k \leqslant N-3$, because the dimensional restrictions they impose follow from the same set of physical quasiprimary states that are used afterwards to eliminate the non-invariant terms of the $\left.\mathscr{F}_{11}\right|_{k}$ sector. Note that even without any reference to the Lie bracket, the Virasoro generators play a distinguished role as an organizing principle for the physical states. The Fock space projections of the Virasoro monomials in $\mathscr{H}_{11}$ have a maximal staggering in their [a] powers, which allows the deduction of an equality in (29). From an arbitrary basis of $\hat{\mathscr{H}}_{11}$ one could only infer inequalities for the $P(\leqslant k), k \leqslant N$. The first point guarantees, in particular, that the recursion can be started. One easily checks that $L_{-2}^{N / 2}$ and $L_{-4}^{(N-2) / 2} \simeq L_{-4} L_{-3} L_{-2}^{(N-2) / 2}$ are the only physical quasiprimary states containing $[a]^{N-3}$; i.e. $P(\leqslant N-3)=2$. In contrast, for $N$ odd, one has $L_{-2}^{(N-3) / 2} L_{-3} \simeq 0$ and $L_{-2}^{(N-5) / 2} L_{-5} \simeq L_{-4} L_{-3} L_{-2}^{(N-7) / 2} \simeq L_{-3}^{3} L_{-2}^{(N-9) / 2}$ is the only physical quasiprimary state containing $[a]^{N-4}$, so that the first step of the recursion fails. From the Jacobi identity, the absence of conservation laws at odd grades implies directly that the conserved charges $\oint \mathrm{d} z P_{N}(z)$ for $N$ even are mutually commuting.

In summary, we can picture the situation in a flow diagram (fig. 1). The test $P(\leqslant N-2) \geqslant 2$ rules out $N$ odd from the beginning. For $N$ even, there are two non-trivial steps in each cycle. The first includes that the calculation of the new representatives in $\left.\tilde{\mathscr{F}}_{11}\right|_{p \leqslant k}$ is actually possible, because of the above dimensional argument. A necessary condition for this dimensional argument to work is that $C(\leqslant k) \geqslant P(\leqslant k)$. The second non-trivial step of the cycle is that this is just guaranteed by the preceeding recursion step. Thus, given the numbers $P(\leqslant k)$, 


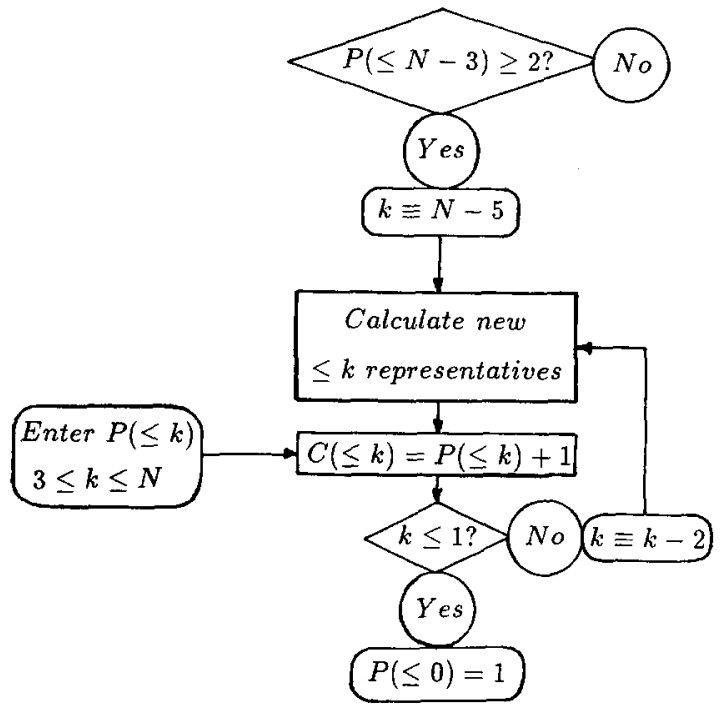

Fig. 1. Flow diagram for recursion procedure.

$3 \leqslant k \leqslant N$, the algorithm will for $N$ even inevitably run until $P(\leqslant 0)=1$, establishing the existence of a conservation law at grade $N$ with unit multiplicity.

As mentioned in the introduction, a similar line of argument can be followed in the case of the $\mathrm{Sl}$ (3)-extended DF model. It turns out that the closure condition for the $\mathscr{W}(\mathrm{Sl}(3)$ ) algebra is not needed. Again, the conformal generators serve mainly as an organizing principle for the physical sector of the Fock space. Thus, there appears to be no principle obstruction to the generalization to arbitrary Lie groups, although the complexity will increase quickly with the rank of the group.

I like to thank H. Nicolai and H.J. de Vega for some useful discussions related to this work. Financial support by Deutsches Elektronen Synchrotron DESY is gratefully acknowledged.

\section{References}

[1] A.B. Zamolodchikov, JETP Lett. 46 (1987) 161.

[2] A.B. Zamolodchikov, Sov. J. Nucl. Phys. 46 (1987) 1090.

[3] A.B. Zamolodchikov, Intern. J. Mod. Phys. A 3 (1988) 743.

[4] A.W.W. Ludwig and J.L. Cardy, Nucl. Phys. B 285 [FS19] (1987) 687.

[5] A.B. Zamolodchikov, Integrable field theory from conformal field theory, Proc. Taganuchi Conf. on Integrable models (1988).

[6] N. Reshetikhin and F. Smirnov, Hidden quantum group symmetry and integrable perturbations of conformal field theories, preprint Harvard HUTMP 89/B246;

D. Bernard and A. LeClair, Residual quantum symmetries of the restricted sine-Gordon theories, preprint Cornell 90/974;

T. Eguchi and S.K. Yang, Sine-Gordon theory at Rational values of the coupling constant and minimal conformal models, preprint Tokyo UT-89-554.

[7] A. Capelli and J.L. Latorre, Perturbation theory of higher-spin converved currents off-critically, preprint Copenhagen NBI-HE-8954 ;

B.A. Kupershmidt and P. Mathieu, Phys. Lett. B 227 (1989) 245.

[8] G. Felder, J. Fröhlich and G. Keller, Commun. Math. Phys. 124 (1989) 647.

[9] A.A. Belavin, A.M. Polyakov and A.M. Zamolodchikov, Nucl. Phys. B 241 (1984) 333.

[10] V.A. Fateev and A.B. Zamolodchikov, Nucl. Phys. B 280 [FS18] (1987) 644;

V.A. Fateev and S. Lykyanov, Intern. J. Mod. Phys. A 3 (1988) 507. 
[11] A. Bilal, Fusion and braiding in extended conformal theories, preprint CERN-TH-5403/89.

[12] in preparation.

[13] B.L. Feigin and D.B. Fuks, Funct. Anal. Appl. 16 (1982) 114; 17 (1983) 241;

C. Thorn, Nucl. Phys. B 248 (1984) 551;

Vl.S. Dotsenko and V.A. Fateev, Nucl. Phys. B 240 [FS12] (1984) 312; B 251 [FS13] (1985) 691;

M. Kato and S. Matsuda, Null field construction in conformal and superconformal algebras, Proc. Kyoto Symp. on Conformal field theory and solvable lattice models.

[14] R. Sasaki and I. Yamanaka, Virasoro algebra, vertex operators, quantum sine-Gordon and solvable quantum field theories, Proc. Kyoto Symp. on Conformal field theory and solvable lattice models (1986);

T. Eguchi and S.K. Yang, Phys. Lett. B 224 (1989) 373. 